\title{
openheart Evidence from randomised controlled trials did not support the introduction of dietary fat guidelines in 1977 and 1983: a systematic review and meta-analysis
}

\author{
Zoë Harcombe, ${ }^{1}$ Julien S Baker, ${ }^{1}$ Stephen Mark Cooper, ${ }^{2}$ Bruce Davies, ${ }^{3}$ \\ Nicholas Sculthorpe, ${ }^{1}$ James J DiNicolantonio, ${ }^{4}$ Fergal Grace ${ }^{1}$
}

To cite: Harcombe Z, Baker JS, Cooper SM, et al. Evidence from randomised controlled trials did not support the introduction of dietary fat guidelines in 1977 and 1983: a systematic review and meta-analysis. Open Heart 2015;2:e000196. doi:10.1136/openhrt-2014000196

Received 18 September 2014 Revised 26 November 2014 Accepted 2 December 2014

CrossMark

\footnotetext{
${ }^{1}$ Institute of Clinical Exercise and Health Science,

University of the West of Scotland, Hamilton, Lanarkshire, UK

${ }^{2}$ Cardiff School of Sport, Cardiff Metropolitan University, Cardiff, UK ${ }^{3}$ University of South Wales, Pontypridd, UK

${ }^{4}$ Saint Luke's Mid America Heart Institute, Kansas City, Missouri, USA
}

Correspondence to Zoë Harcombe;

Zoe.Harcombe@uws.ac.uk

\section{ABSTRACT}

Objectives: National dietary guidelines were introduced in 1977 and 1983, by the US and UK governments, respectively, with the ambition of reducing coronary heart disease (CHD) by reducing fat intake. To date, no analysis of the evidence base for these recommendations has been undertaken. The present study examines the evidence from randomised controlled trials (RCTs) available to the US and UK regulatory committees at their respective points of implementation.

Methods: A systematic review and meta-analysis were undertaken of RCTs, published prior to 1983, which examined the relationship between dietary fat, serum cholesterol and the development of CHD.

Results: 2467 males participated in six dietary trials: five secondary prevention studies and one including healthy participants. There were 370 deaths from allcause mortality in the intervention and control groups. The risk ratio (RR) from meta-analysis was 0.996 (95\% Cl 0.865 to 1.147). There were 207 and 216 deaths from $\mathrm{CHD}$ in the intervention and control groups, respectively. The RR was $0.989(95 \% \mathrm{Cl} 0.784$ to 1.247$)$. There were no differences in all-cause mortality and non-significant differences in CHD mortality, resulting from the dietary interventions. The reductions in mean serum cholesterol levels were significantly higher in the intervention groups; this did not result in significant differences in CHD or all-cause mortality. Government dietary fat recommendations were untested in any trial prior to being introduced. Conclusions: Dietary recommendations were introduced for 220 million US and 56 million UK citizens by 1983 , in the absence of supporting evidence from RCTs.

\section{INTRODUCTION}

US public health dietary advice was announced by the Select Committee on Nutrition and Human needs in $1977^{1}$ and was followed by UK public health dietary

\section{KEY MESSAGES}

What is already known about this subject?

- Dietary recommendations were introduced in the US (1977) and in the UK (1983) to (1) reduce overall fat consumption to $30 \%$ of total energy intake and (2) reduce saturated fat consumption to $10 \%$ of total energy intake.

\section{What does this study add?}

- No randomised controlled trial (RCT) had tested government dietary fat recommendations before their introduction. Recommendations were made for 276 million people following secondary studies of 2467 males, which reported identical all-cause mortality. RCT evidence did not support the introduction of dietary fat guidelines.

How might this impact on clinical practice?

- Clinicians may be more questioning of dietary guidelines, less accepting of low-fat advice (concomitantly high carbohydrate) and more engaged in nutritional discussions about the role of food in health.

advice issued by the National Advisory Committee on Nutritional Education in $1983 .^{2}$ The dietary recommendations in both cases focused on reducing dietary fat intake; specifically to (1) reduce overall fat consumption to $30 \%$ of total energy intake and (2) reduce saturated fat consumption to $10 \%$ of total energy intake.

The recommendations were an attempt to address the incidence of coronary heart disease (CHD). Both documents acknowledged that the evidence was not conclusive. Hegsted's introduction to the Dietary Goals for the US noted "there will undoubtedly be many people who will say we have not proven our point."1 The UK publication referred to "a strong consensus of opinion." 2 
The evidence available to dietary committees at that time comprised epidemiological studies and randomised controlled trials (RCTs). The most comprehensive population study undertaken was the Seven Countries Study by Keys. ${ }^{3}$ This reported that CHD 'tended to be related' to serum cholesterol values and that, these in turn 'tended to be related' to the proportion of calories provided by saturated fats in the diet. ${ }^{4}$ Keys acknowledged that epidemiological studies could reveal relationships, not causation. ${ }^{3}$ RCTs provide the best evidence. ${ }^{5}$

While the UK nutritional guidelines ${ }^{2}$ made reference to the Seven Countries Study, the US committee document ${ }^{1}$ did not. Neither publication made reference to any of the RCTs available at that time. However, the US Committee report reported data from the non-randomised, cross-over trial, the Finnish Mental Hospital Study. ${ }^{6} 7$

Although a number of reviews of RCTs have been undertaken, ${ }^{8-10}$ no review has examined the RCT evidence available at the time dietary fat guidelines were introduced. Furthermore, these guidelines have not been changed since they had been announced; correspondingly, the validity of their evidence base remains relevant.

This systematic review and meta-analysis will assess if the published RCTs available to the dietary committees supported their recommendations on dietary fat. With this in mind, we hypothesised that RCT evidence available to the dietary committees at the time of issuing recommendations did not support the contention that reducing dietary fat intake would contribute to a reduction in CHD risk or related mortality.

\section{METHODS}

A systematic review and meta-analysis was conducted in accordance with the PRISMA guidelines. ${ }^{11}$

\section{Search strategy}

A search was undertaken to identify RCTs that examined the relationship between dietary fat, serum cholesterol and mortality. Exclusion criteria were: study being observational; non-randomised and/or multifactorial in design. Inclusion criteria were: randomised dietary intervention studies; study hypothesis relating to a reduction or modification of dietary fat or cholesterol; participants were human adults; study was a minimum of 1 year in duration; data on all-cause mortality, CHD mortality and cholesterol measurements were available.

Searches were performed of the literature to 1983 using MEDLINE and the Cochrane Library. The AMED, CAB abstracts, CINAHL, EMBASE, HMIC and SIGLE (grey literature sources) were not relied on, as their periods covered were not compatible: from 1985, 1973, 1981, 1980, 1983 and 1992, respectively. ${ }^{12}{ }^{13}$ (figure 1).

\section{Selection of studies}

Of the 98 identified articles, 80 were rejected on review of the title and abstract. The remaining 18 papers covered 8 trials, once duplication was resolved. Six RCTs met the inclusion criteria: Rose Corn Oil Trial; ${ }^{14}$ Research Committee Low-fat Diet; ${ }^{15}$ MRC Soya-bean Oil ${ }^{16}$ LA Veterans Study; ${ }^{17}$ Oslo Diet Heart Study ${ }^{18}{ }^{19}$; and The Sydney Diet Heart Study. ${ }^{20}$ The Anti-Coronary club trial ${ }^{21}$ and The Finnish Mental Hospital Study ${ }^{6} 7$ were excluded, as they were not randomised. The Finnish study was also a cross-over trial. This is not appropriate for the examination of a long-term mortality, as deaths in the second phase may be due to conditions imposed during the initial phase. Inclusion criteria are in agreement with previous literature. ${ }^{9}$

To ascertain the validity of eligible randomised trials, a pair of reviewers ( $\mathrm{ZH}$ and $\mathrm{FG}$ ) worked independently to determine which studies met the inclusion criteria. The same six were agreed on. Risk of bias was further assessed using the PEDro scale for the relative quality of studies. ${ }^{22}$ Additionally, the meta-analyses for all-cause mortality (figure 2) and CHD deaths (figure 3) were tested for sensitivity analysis of the exclusion of any one study.

\section{Data extraction}

Table 1 details data extraction including: study name, duration, year of publication and confirmation of study design; participant characteristics; details of intervention and comparison diet; and outcomes relating to all-cause mortality, CHD-related deaths and changes in mean serum cholesterol levels. Where a study contained more than one intervention, both were included. ${ }^{14}$

\section{Statistical analysis}

The overall pooled effect was calculated using random effects meta-analysis. Heterogeneity was evaluated using the Q-value, $\mathrm{I}^{2}$ and $\mathrm{T}^{2}$ calculations. Funnel plot methodology $^{24}$ and Egger's regression intercept ${ }^{23}{ }^{24}$ have been calculated, noting the caution for analysing publication bias using funnel plot asymmetry where the meta-analysis has fewer than 10 studies. $^{25}$ Analyses were performed using Comprehensive Meta-Analysis. ${ }^{26}$

\section{RESULTS \\ Participants and study design}

The identified RCTs included a total of 740 deaths and 423 deaths from CHD among 2467 male participants (table 1). All but one trial exclusively studied secondary prevention participants. The LA Veterans $\operatorname{Study}^{17}$ comprised one-fifth secondary participants and four-fifths primary participants.

All trials were parallel and randomised. Two were blinded for outcome assessment. ${ }^{15}{ }^{16}$ Two were open, with no blinding on either side. ${ }^{19}{ }^{20}$ The LA Veterans study ${ }^{17}$ was reported as double blinded, but the dietary changes were so substantial that this seems implausible (egg consumption quantified, vegetable oils added and animal fats restricted). Rose $e t a l^{14}$ was reported as blinded to intervention participants for the type of oil, but not blinded for outcome assessment. 


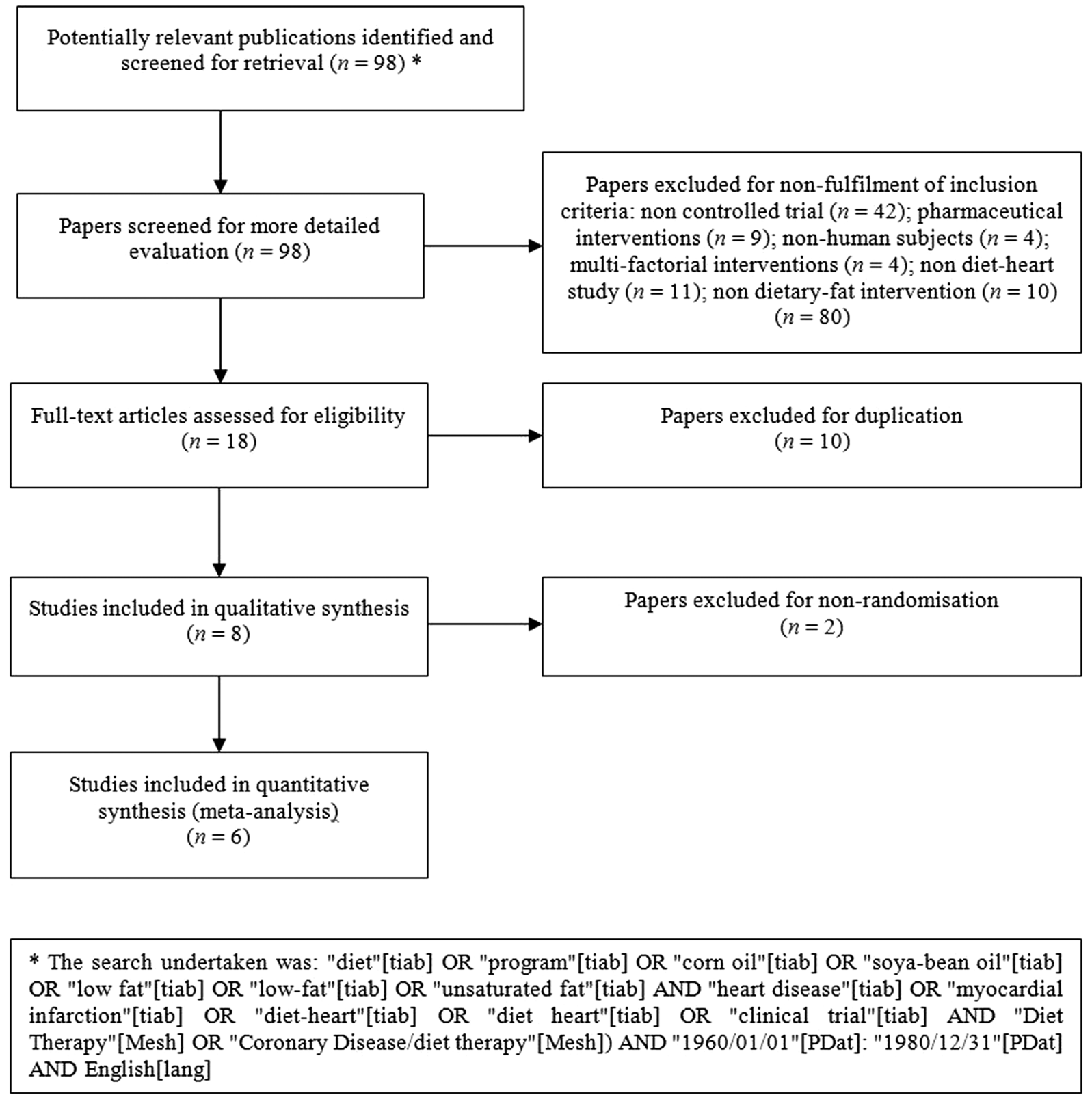

Figure 1 Summary of systematic review profile. ${ }^{11}$

The mean duration of the six trials was $5.4 \pm 3.5$ years. The weighted mean (person years by people) was 6.5 \pm 1 years.

Quality scores were moderate and relatively homogenous: all trials had quality scores of 4 or 5 using the PEDro scale. ${ }^{22}$ The meta-analyses for all-cause mortality (figure 2) and CHD deaths (figure 3) were tested for sensitivity analysis of the exclusion of any one study. There were no circumstances in which the exclusion of any one study made the overall effect size significant.

Statistical evidence for substantial between study heterogeneity was not found. For all deaths, the Q-value was 7.115 ( $6^{\circ}$ of freedom). $\mathrm{I}^{2}$ was 15.676 and $\mathrm{T}^{2}$ was 0.006 . For CHD deaths, the Q-value was 8.649 ( $6^{\circ}$ of freedom). $\mathrm{I}^{2}$ was 30.632 and $\mathrm{T}^{2}$ was 0.028 .
Visual inspection of the funnel plots revealed that none of the studies lay outside the SE funnel for the meta-analysis of all deaths or CHD deaths. The two, small, Rose $e t a l^{14}$ studies produced asymmetry on the lower right hand side of the funnels. Egger's regression test confirmed some asymmetry, noting the caution for the small number of studies. ${ }^{25}$ The Egger's regression intercept was 1.029 (95\% CI two-tailed, -0.433 to 2.492) (one-tailed $\mathrm{p}=0.065$; two-tailed $\mathrm{p}=0.130$ ) for the all deaths meta-analysis and 1.554 (95\% CI two-tailed, -0.013 to 3.121) (one-tailed $\mathrm{p}=0.025$; two-tailed $\mathrm{p}=0.051$ ) for CHD deaths.

\section{Interventions and comparisons}

Five of the six RCTs did not examine either a total fat consumption of $30 \%$, or a saturated fat consumption of 
Dietary Intervention \& All Deaths

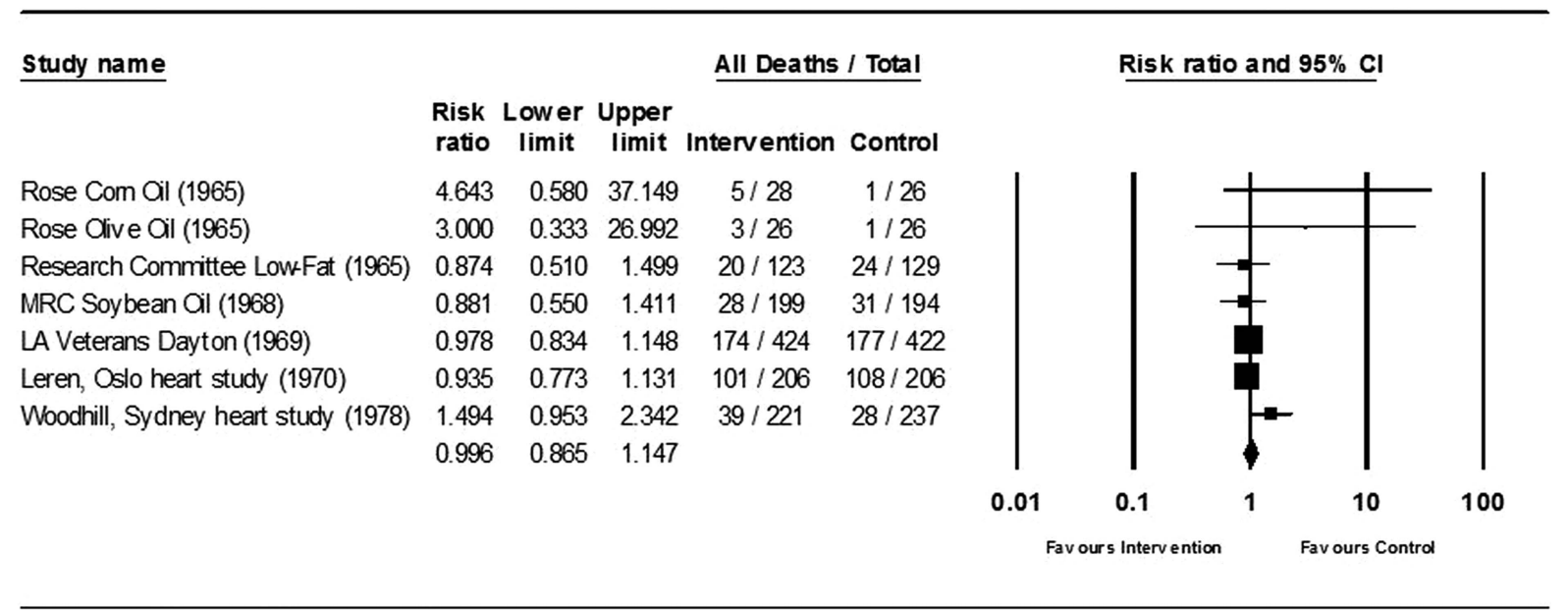

Meta Analysis Random Effects Method

Figure 2 Estimates of total mortality ( $95 \% \mathrm{Cls})$ from meta-analysis.

$10 \%$, of energy intake. The trials examined: the administration of vegetable oil; ${ }^{14} 161719$ the replacement of saturated fats with vegetable oil ${ }^{1617} 19$ and an approximate $20 \%$ fat diet. ${ }^{15}$ A single RCT $^{20}$ examined the consequence of a $10 \%$ saturated fat diet and reported a higher incidence of all-cause mortality and CHD deaths in the intervention group.

\section{Outcomes: all-cause mortality}

Across six studies containing seven dietary interventions ${ }^{14}$ involving 1227 people in the intervention groups and 1240 people in the control groups, there were 370 deaths in the intervention and control groups. The allcause mortality was $30.2 \%$ in the intervention groups and $29.8 \%$ in the control groups.
The mean death rate was high reflecting the fact that these were secondary prevention studies, except for the combined primary and secondary prevention LA Veterans study. Unsurprisingly death rates were higher in the longer term studies. The lowest death rate was observed in the control group of Rose et al. ${ }^{14}$

In the meta-analysis of all-cause mortality, the LA Veterans study $^{17}$ carried the greatest weight, $41.71 \%$, (figure 2 random effects methodology). The corn and olive oil interventions had negligible impact on the overall effect, with weights of $0.46 \%$ and $0.41 \%$, respectively. ${ }^{14}$ The risk ratio (RR) for all seven studies was 0.996 (95\% CI 0.865 to 1.147 ). The overall effect measurement lies on the line of no effect. There was no statistically significant relationship between dietary interventions and all-cause mortality.

\section{Dietary Intervention \& Heart Deaths}

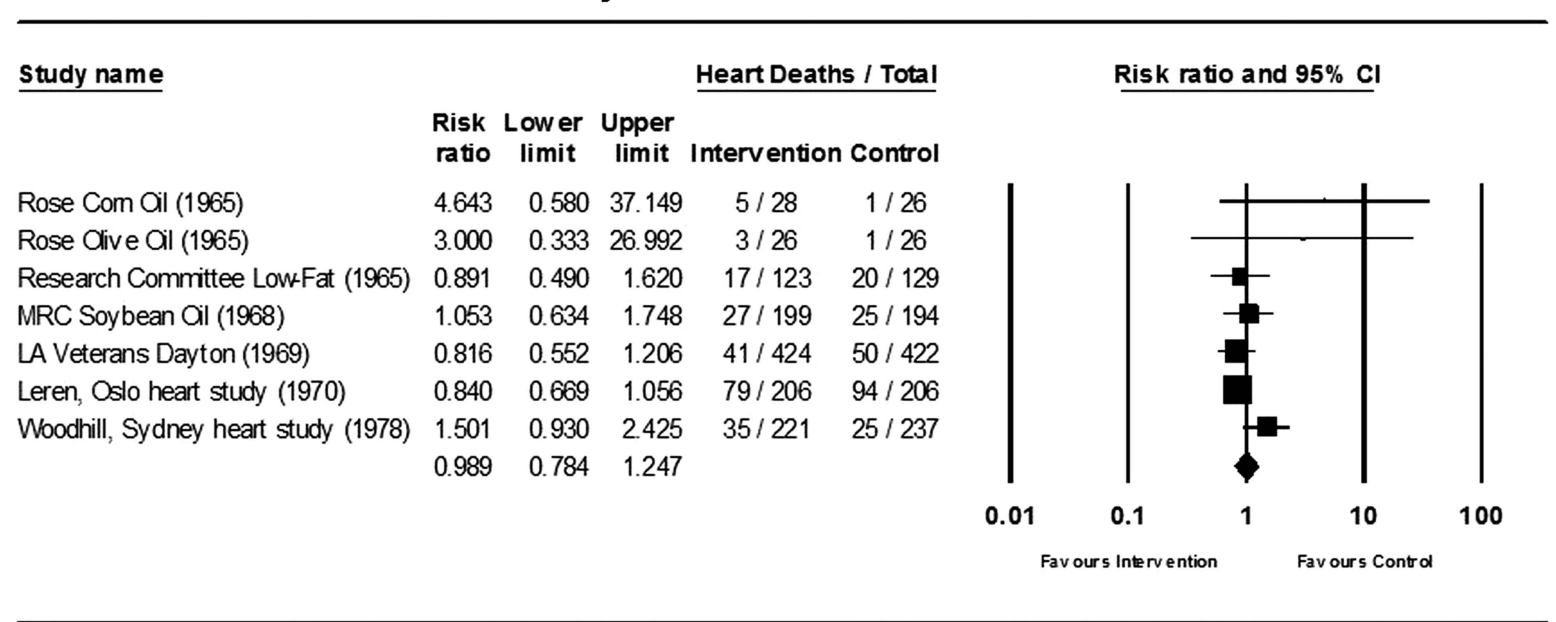

Meta Analysis Random Effects Method

Figure 3 Estimates of CHD mortality $(95 \% \mathrm{Cls})$ from meta-analysis. 


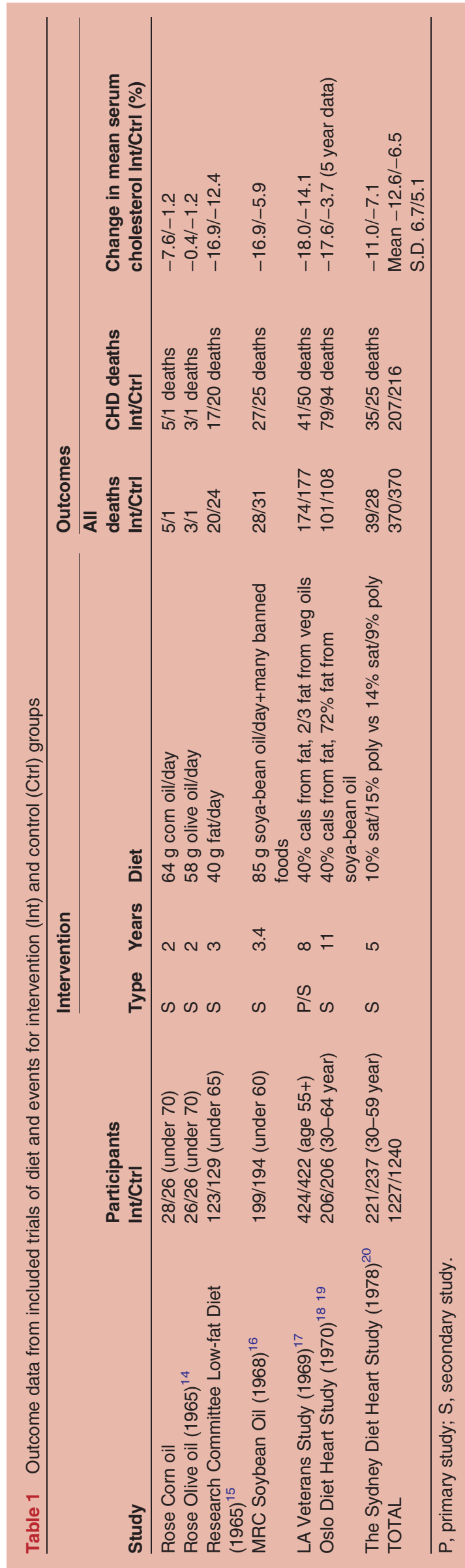

\section{CHD mortality}

The seven interventions recorded 207 deaths from CHD in the intervention groups and 216 in the control groups. The forest plot for the dietary interventions and deaths from CHD produced the meta-analysis is shown in figure 3 (random effects methodology).

In the meta-analysis of CHD mortality, the Leren Oslo study $^{19}$ carried the greatest weight, $34.16 \%$, (figure 3 random effects methodology). The corn and olive oil interventions carried the least weight with $1.22 \%$ and $1.09 \%$, respectively. ${ }^{14}$ The RR for all seven studies was 0.989 (95\% CI 0.784 to 1.247). The overall effect measurement lies on the line of no effect. There was no statistically significant relationship between dietary interventions and heart deaths.

\section{Significance reported by the studies}

Three studies ${ }^{15-17}$ and the olive oil ${ }^{14}$ intervention reported no significant differences in deaths. The corn oil deaths were reported as significantly different, in favour of the control group $(0.1>\mathrm{p}>0.05) .{ }^{14}$ Leren $^{19}$ reported that the difference in all-cause mortality was not statistically significant $(\mathrm{p}=0.35)$. The total CHD mortality was 79 out of 206 men in the diet group and 94 out of 206 men in the control group $(p=0.097)$. Woodhill $e t a l^{20}$ recorded 39 deaths in the intervention group and 28 in the control group. There were 35 deaths from CHD in the intervention group and 25 in the control group. These were described as significant, but not endorsed by statistical analysis.

\section{Serum cholesterol levels}

Mean serum cholesterol levels fell in all groups: control and intervention. The standardised mean difference in serum cholesterol levels, for the six trials (seven interventions) combined, was $-12.6 \% \pm 6.7 \%$ for the intervention groups and $-6.5 \% \pm 5.1 \%$ for the control groups (table 1). The effect size was 1.04 .

\section{DISCUSSION}

The main findings of the present meta-analysis of the six RCTs available at the time of issuing dietary guidelines in the US and UK indicate that all-cause mortality was identical at 370 in the intervention and control groups. There was no statistically significant difference in deaths from CHD. The reductions in mean serum cholesterol levels were significantly higher in the intervention groups; this did not result in significant differences in CHD or all-cause mortality.

It is a widely held view that reductions in cholesterol are healthful per se. The original RCTs did not find any relationship between dietary fat intake and deaths from CHD or all-causes, despite significant reductions in cholesterol levels in the intervention and control groups. This undermines the role of serum cholesterol levels as an intermediary to the development of $\mathrm{CHD}$ and contravenes the 
theory that reducing dietary fat generally and saturated fat particularly potentiates a reduction in CHD.

There are some important design limitations among the available RCTs. The LA Veterans study ${ }^{17}$ provided meals in a contained environment, but was undermined by open enrolment, allowing participants to leave and join. The other five RCTs relied on dietary advice, with meetings and periodical dietary analysis to monitor adherence. Three of these studies audited outcomes and the data extracted in table 1 recorded actual, not target, dietary intake. ${ }^{14} 19 \quad 20$ A number of studies impaired assessment of one intervention (administering oils) by adding other dietary restrictions. ${ }^{16} 1719$

The LA Veterans study ${ }^{17}$ recorded the lowest RR for CHD deaths for the intervention group: 0.816 (figure $3)$. However, there were important differences in the groups at study entry. The intervention group had 12 octogenarians, compared with 21 in the control group. Eleven per cent of the experiment group were heavy smokers (more than one pack a day) compared with $17 \%$ of the control group.

Woodhill et $a t^{20}$ made an important observation that men who have suffered an myocardial infarction (MI) subsequently make multiple lifestyle changes (weight loss, smoking cessation, increase in physical activity, for example), which makes them a poor group for testing the lipid hypothesis. In this respect, the reporting of cholesterol decreases in control and intervention groups supports the observation that multiple lifestyle changes are made.

Studies of the time report weight, not body mass. Weight changes were not recorded in two studies. ${ }^{14} 19$ The two studies noted no significant weight change in intervention or control groups. ${ }^{16}{ }^{17}$ The Research Committee study ${ }^{15}$ reported mean weight loss as $7.5 \%$ in the intervention group and $4.8 \%$ in the control group. Woodhill $e t a l^{20}$ reported a mean weight loss of $6.5 \%$ and $6 \%$ in the intervention and control groups, respectively.

The phytosterol content of vegetable oils could explain reductions in cholesterol levels with no concomitant reductions in deaths. ${ }^{27}$

A limitation of the present review and meta-analysis relates to the detailed information in the original studies in order to determine the saturated, monounsaturated and polyunsaturated content of the control and intervention diets. Woodhill $e t a l^{20}$ was the only study to detail the composition of the three fats in the intervention and control diet; a $10 \%$ saturated fat intake being the intervention goal. Leren ${ }^{19}$ documented the intervention diet as $40 \%$ of total calories as fat; $8.3 \%$ of total calories as saturated fat and a polyunsaturated to saturated fat ratio of 2.4:1. No comparable measures were given for the control diet in this study. Other studies recorded total fat intake as a percentage of calories, but not individual fat composition. Consequently, deductions about the relationship between different fats and serum cholesterol levels cannot be made.
Deductions can be made about the dietary interventions and mortality from all-causes and CHD. The Rose et $a l^{14}$ interventions most notably favour the control in both forest plots, but the wide CIs render these, as with all the studies, non-significant.

Only one study made a positive claim for its intervention after 5 years ${ }^{18}$ and subsequently, this was moderated. ${ }^{19}$ Rose $e t a l^{14}$ warned of possible harm by administering corn oil. The Research Committee ${ }^{15}$ concluded "A low-fat diet has no place in the treatment of myocardial infarction" (p504). The MRC Soya-bean oil ${ }^{16}$ intervention found no evidence that MI relapse would be materially affected by unsaturated fat in the diet. The LA Veterans study ${ }^{17}$ reported that total longevity was not affected and expressed concern about unknown toxicity of their intervention. Woodhill et $a l^{20}$ noted that survival was significantly better in the control than the diet group.

In the absence of epidemiological evidence from whole-populations, large-scale RCTs of longer duration (with adequate follow-up), which accounted for known confounding variables and included primary participants of both males and females, may have supported the introduction of dietary fat guidelines in 1977 and 1983. However, this opportunity expired when universal pharmacological treatment became the accepted norm.

From the literature available, it is clear that at the time dietary advice was introduced, 2467 men had been observed in RCTs. No women had been studied; no primary prevention study had been undertaken; no RCT had tested the dietary fat recommendations; no RCT concluded that dietary guidelines should be introduced. It seems incomprehensible that dietary advice was introduced for 220 million Americans ${ }^{28}$ and 56 million UK citizens, ${ }^{29}$ given the contrary results from a small number of unhealthy men.

An exchange between Dr Robert Olson of St Louis University and Senator George McGovern, chair of the Dietary Committee, was recorded in July $1977 .{ }^{30}$ Olson said "I pleaded in my report and will plead again orally here for more research on the problem before we make announcements to the American public." McGovern replied "Senators don't have the luxury that the research scientist does of waiting until every last shred of evidence is in".

There was best practice, randomised controlled trial, evidence available to the dietary committees, which was not considered and should have been. The results of the present meta-analysis support the hypothesis that the available RCTs did not support the introduction of dietary fat recommendations in order to reduce CHD risk or related mortality.

Two recent publications have questioned the alleged relationship between saturated fat and CHD and called for dietary guidelines to be reconsidered. ${ }^{31} 32$

The present review concludes that dietary advice not merely needs review; it should not have been introduced.

Contributors $\mathrm{ZH}$ conceived of the study and was the major contributor to data extraction, writing of the manuscript and the meta-analysis. FG was 
involved in data extraction, writing of the manuscript and the meta-analysis. BD was involved in writing of the manuscript. NS was involved in the meta-analysis. All authors contributed to the manuscript. All authors were involved in critical evaluation of content.

Competing interests None.

Provenance and peer review Not commissioned; externally peer reviewed.

Data sharing statement The PRISMA checklist can be shared with the publication. The full list of papers from the first search return can be obtained from the corresponding author (excel file). The Comprehensive Meta Analysis files can be shared with other users of the CMA package-available from the corresponding author.

Open Access This is an Open Access article distributed in accordance with the Creative Commons Attribution Non Commercial (CC BY-NC 4.0) license, which permits others to distribute, remix, adapt, build upon this work noncommercially, and license their derivative works on different terms, provided the original work is properly cited and the use is non-commercial. See: http:// creativecommons.org/licenses/by-nc/4.0/

\section{REFERENCES}

1. Select Committee on Nutrition and Human Needs. Dietary goals for the United States. 1st edn. Washington: US Govt Print Off, 1977.

2. National Advisory Committee on Nutritional Education (NACNE). A discussion paper on proposals for nutritional guidelines for health education in Britain, 1983.

3. Keys A. Coronary heart disease in seven countries. I. The study program and objectives. Circulation 1970;41(4 Suppl):I1-8.

4. Keys A. Coronary heart disease in seven countries. Summary. Circulation 1970;41(4 Suppl):I186-95.

5. Barton S. Which clinical studies provide the best evidence? The best RCT still trumps the best observational study. BMJ 2000;321:255-6.

6. Turpeinen $\mathrm{O}$, Karvonen MJ, Pekkarinen $\mathrm{M}$, et al. Dietary prevention of coronary heart disease: the Finnish Mental Hospital Study. Int J Epidemiol 1979;8:99-118.

7. Miettinen M, Turpeinen $\mathrm{O}$, Karvonen $\mathrm{MJ}$, et al. Dietary prevention of coronary heart disease in women: the Finnish mental hospital study. Int J Epidemiol 1983;12:17-25.

8. Truswell AS. Review of dietary intervention studies: effect on coronary events and on total mortality. Aust N Z J Med 1994;24:98-106.

9. Hooper L, Summerbell CD, Higgins JPT, et al. Dietary fat intake and prevention of cardiovascular disease: systematic review. BMJ 2001;322:757-63.

10. Mozaffarian D, Micha R, Wallace S. Effects on coronary heart disease of increasing polyunsaturated fat in place of saturated fat: a systematic review and meta-analysis of randomized controlled trials. PLoS Med 2010;7:e1000252.

11. Moher D, Liberati A, Tetzlaff J, et al. Preferred reporting items for systematic reviews and meta-analyses: the PRISMA statement. J Clin Epidemiol 2009;62:1006-12.
12. National Institute of Clinical Excellence (NICE). Journals and databases. Secondary Journals and databases. 2014. http://www. library.nhs.uk/help/resource

13. SIGLE System for Information on Grey Literature in Europe. Grey Literature Network Service. Secondary Grey Literature Network Service Founded. 1992. http://www.opengrey.eu/about/greyliterature

14. Rose GA, Thomson WB, Williams RT. Corn oil in treatment of ischaemic heart disease. BMJ 1965;1:1531-3.

15. Research Committee. Low-fat diet in myocardial infarction: a controlled trial. Lancet 1965;2:501-4.

16. Medical Research Council. Controlled trial of soya-bean oil in myocardial infarction: Report of a research committee to the Medical Research Council. Lancet 1968;292:693-700.

17. Dayton S, Pearce ML, Hashomoto S, et al. A Controlled clinical trial of a diet high in unsaturated fat in preventing complications of atherosclerosis. Circulation 1969;40:(1S2):II-1-II-63.

18. Leren $P$. The effect of plasma-cholesterol-lowering diet in male survivors of myocardial infarction. A controlled clinical trial. Bull N Y Acad Med 1968;44:1012-20.

19. Leren P. The Oslo Diet-Heart Study. Circulation 1970;42:935-42.

20. Woodhill JM, Palmer AJ, Leelarthaepin B, et al. Low fat, low cholesterol diet in secondary prevention of coronary heart disease. Adv Exp Med Biol 1978;109:317-30.

21. Christakis G, Rinzler SH, Archer M, et al. Effect of the anti-coronary club program on coronary heart disease risk-factor status. JAMA 1966;198:597-604

22. de Morton NA. The PEDro scale is a valid measure of the methodological quality of clinical trials: a demographic study. Aust $J$ Physiother 2009;55:129-33.

23. Egger M, Smith GD, Schneider M, et al. Bias in meta-analysis detected by a simple, graphical test. BMJ 1997;315:629-34.

24. Sterne JAC, Sutton AJ, loannidis JPA, et al. Recommendations for examining and interpreting funnel plot asymmetry in meta-analyses of randomised controlled trials. BMJ 2011;343:d4002.

25. Higgins JPT, Green S. Cochrane handbook for systematic reviews of interventions. The Cochrane Collaboration, 2011; Version 5.1.0.

26. Comprehensive Meta Analysis Version 2 [program]. 2 version. Englewood, NJ: Biostat 2005

27. Bresson J-L. Scientific Opinion of the Panel on Dietetic Products Nutrition and Allergies on a request from Unilever PLC/NV on Plant Sterols and lower/reduced blood cholesterol, reduced the risk of (coronary) heart disease. EFSA J 2008;781:1-12.

28. United Stated Census Bureau. U.S. Resident Population, 1977

29. Office for National Statistics. Population Estimates for UK, England and Wales, Scotland and Northern Ireland, Mid-1982 to Mid-1990 Population Estimates for England \& Wales. Office for National Statistics, 2003.

30. CBS News. Exchange between Dr Robert Olson and Senator George McGovern from The United States Senate Select Committee on Nutrition and Human Needs. Washington, 26 July 1977.

31. DiNicolantonio JJ. The cardiometabolic consequences of replacing saturated fats with carbohydrates or $\Omega-6$ polyunsaturated fats: Do the dietary guidelines have it wrong? Open Heart 2014;1:e000032.

32. Chowdhury R, Warnakula S, Kunutsor S, et al. Association of Dietary, Circulating, and Supplement Fatty Acids With Coronary Risk: A Systematic Review and Meta-analysis. Ann Intern Med 2014;160:398-406. 


\section{Correction}

Harcombe Z, Baker JS, Cooper SM, et al. Evidence from randomised controlled trials did not support the introduction of dietary fat guidelines in 1977 and 1983: a systematic review and meta-analysis. Open Heart 2015;2:e000196. doi:10.1136/openhrt-2014-000196

Authors' statement:

"The authors confirm that they have no conflicts of interest regarding the funding or outcomes of the above study. The study was not funded (either in full or in part) by any private, charitable or government institutions. No financial payments were received for any aspect of the above work. There are no financial relationships with any third party entities relating to the above work, nor are there any patents (either submitted or planned) relating to the study.

In addition to the above, Mrs Harcombe receives income from writing and from The Harcombe Diet Co. and from Columbus Publishing, in addition to the national minimum doctoral stipend from the University of the West of Scotland".

\section{Editor's comment:}

"Following comments from readers, and post-publication discussions within our editorial team, the authors of this paper were asked to update their competing interest statement. The potential competing interests relate to one of the authors of the article, Mrs Harcombe, who has previously published books on diet and nutrition, and is also a co-director of a company that gives dietary advice (The Harcombe Diet Co.) and co-director of a publishing company (Columbus Publishing) that publishes books on diet and nutrition.

At the time of submission of the manuscript, the authors did not declare these competing interests because they did not perceive them as directly relevant, as many authors of research articles have previously written books or given public advice without declaring these activities in their research articles.

The editorial team of Open Heart firmly believes that to make the best decision on how to interpret the research data, readers need to be aware of any potential competing interests that authors may have. We ask that all authors declare competing interests in their covering letter and in a statement headed "Competing Interests" at the end of their manuscript. For information on what should be declared, we refer our authors to an editorial published by The BMJ: Beyond conflict of interest (BMJ 1998;317:291-2).

In this case, Open Heart feels that the books and companies with which Mrs Harcombe has been involved should have been declared. From our point of view, a competing interest exists when professional judgement concerning a primary interest (such as validity of research) could potentially be influenced by a secondary interest (such as financial gain). Currently, Open Heart restricts requests for competing interest declarations to financial interests only.

While it is not our goal to eliminate competing interests, since they are almost inevitable, and while we would not reject papers purely because authors have a competing interest, we absolutely feel that they need to be declared transparently. There is nothing inherently unethical about a competing interest but it should be acknowledged and openly declared. We appreciate the authors' willingness to update their competing interest statement."

- Pascal Meier, Editor-in-Chief Open Heart.

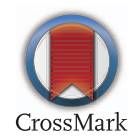

Open Heart 2015;2:e000196. doi:10.1136/openhrt-2014-000196corr1 